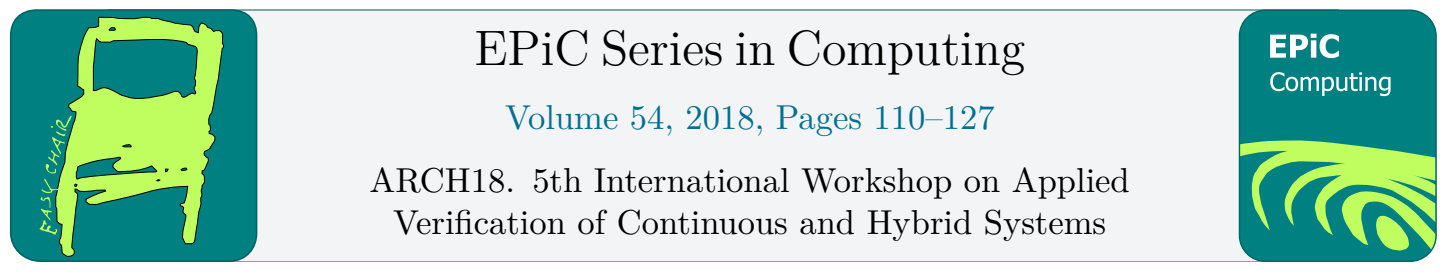

\title{
ARCH-COMP18 Category Report: Hybrid Systems Theorem Proving
}

\author{
Stefan Mitsch ${ }^{1}$, Andrew Sogokon ${ }^{1}$, Yong Kiam Tan ${ }^{1}$, and André Platzer ${ }^{1}$ \\ Hengjun Zhao ${ }^{3}$, Xiangyu $\mathrm{Jin}^{2}$, Shuling Wang ${ }^{2}$, and Naijun Zhan ${ }^{2}$ \\ 1 Computer Science Department, Carnegie Mellon University, Pittsburgh, PA, USA \\ \{smitsch, asogokon, yongkiat, aplatzer\}@cs.cmu.edu \\ 2 State Key Lab of Computer Science, Institute of Software, Chinese Academy of Sciences \\ 3 School of Computer and Information Science, Southwest University, Chongqing, China
}

\begin{abstract}
This paper reports on establishing Hybrid Systems Theorem Proving (HSTP) as a new category in the ARCH-COMP Friendly Competition 2018. The most important characteristic features of the HSTP category are: $i$ ) The flexibility of programming languages as structuring principles for hybrid systems, ii) The unambiguity and precision of program semantics, and iii) The mathematical rigor of logical reasoning principles. The HSTP category especially features many nonlinear and parametric continuous and hybrid systems. Owing to the nature of theorem proving, HSTP is able to accomodate three modes: A) Automatic in which the entire verification is performed fully automatically without any additional input beyond the original hybrid system and its safety specification. $\mathbf{H}$ ) Hints in which select proof hints are provided as part of the input problem specification, allowing users to communicate specific advice about the system such as loop invariants. S) Scripted in which a significant part of the verification is done with dedicated proof scripts or problem-specific proof tactics. This threefold split makes it possible to better identify the sources of scalability and efficiency bottlenecks in hybrid systems theorem proving. The existence of all three categories also makes it easier for new tools with a different focus to participate in the competition, wherever they focus on in the spectrum from fast proof checking all the way to full automation. The types of benchmarks considered and experimental findings are described in this paper as well.
\end{abstract}

\section{Introduction}

This report summarizes the experimental results of the Hybrid Systems Theorem Proving (HSTP) category in the ARCH-COMP18 friendly competition. The benchmark examples in the HSTP competition strive for a large variety in hybrid systems modeling patterns of basic extent to provide a low entry barrier for tools as well as examples at scale to identify opportunities for improving on proof automation, scalability and efficiency. The almost 140 examples in the benchmark competition are grouped into the following categories:

- Hybrid systems design shapes: small-scale examples over a large variety of model shapes to test for prover flexibility. 
- Nonlinear continuous models: test for prover flexibility in terms of generating and proving properties about continuous dynamics.

- Hybrid systems case studies: hybrid systems models and specifications at scale to test for application scalability and efficiency.

In each of these categories, tools can select the degree of automation as follows, depending on their focus in the spectrum from fast proof checking to full proof automation:

(A) Automated: hybrid systems models and specifications are the only input, proofs and counterexamples are produced fully automatically.

(H) Hints: select proof hints (e.g., loop invariants) are provided as part of the specifications.

(S) Scripted: significant parts of the verification is done with dedicated problem-specific scripts or tactics.

All benchmark examples are available at https://github.com/LS-Lab/KeYmaeraX-projects/ tree/master/benchmarks and specified in differential dynamic logic $(\mathrm{d} \mathcal{L})$ [Pla08, Pla17], whose format and ASCII syntax are presented in Section 2. The participating tools are presented in Section 3. An overview of the examples together with the findings from the competition is given in Section 4. To establish further trustworthiness of the results, the tools with which the results have been obtained are available at gitlab.com/goranf/ARCH-COMP.

\section{Problem Format}

All benchmarks in the Hybrid Systems Theorem Proving (HSTP) category are written in differential dynamic logic $(\mathrm{d} \mathcal{L})$ [Pla08, Pla17] which has axioms and an unambiguous semantics available $\left[\mathrm{BRV}^{+}{ }^{17}\right]$ in KeYmaera 3, KeYmaera X, Isabelle/HOL, and Coq. To make it easier for tools to participate in the HSTP category, almost all benchmarks in the HSTP category are differential dynamic logic formulas of the particular safety form

$$
\phi \rightarrow[\alpha] \psi
$$

$\begin{array}{cll}\phi & \text { is a real arithmetic formula describing the initial conditions, } \\ \text { where } & \psi & \text { is a real arithmetic formula describing the postcondition / set of safe states, and }\end{array}$

$\alpha$ is the hybrid system described using hybrid programs as a program notation.

The $\mathrm{d} \mathcal{L}$ formula (1) means that if the system starts in a state satisfying the initial condition $\phi$, then all final states of all possible runs of the hybrid system $\alpha$ satisfy postcondition $\psi$. The operators / statements of hybrid programs are summarized in Table 1. Those of logical formulas in $\mathrm{d} \mathcal{L}$ are summarized in Table 2 . In particular, the hybrid program $\alpha$ contains both the discrete and continuous dynamics of the hybrid system.

An example with a purely continuous system is:

$$
-\frac{4}{5}<x<-\frac{1}{3} \wedge-1 \leq y<0 \rightarrow\left[x^{\prime}=2 x-2 x y, y^{\prime}=2 y-x^{2}+y^{2}\right](x+y \leq 1 \wedge(x \neq 0 \vee y \neq 0))
$$

An example with a trivial hybrid system is:

$$
\left.v \geq 0 \wedge A>0 \wedge b>0 \rightarrow\left[(? v \leq 5 ; a:=A \cup a:=-b) ;\left\{x^{\prime}=v, v^{\prime}=a \& v \geq 0\right\}\right)^{*}\right] v \geq 0
$$

This particular example is completely trivial, because the postcondition $v \geq 0$ directly follows from the evolution domain constraint $v \geq 0$ in the differential equation. But safety properties become more exciting and more challenging when the postcondition is a different one. For example, $x \geq 10$ to say that the position is at least 10 always is much more complicated (and not even true for the above example). 
Table 1: Statements of hybrid programs ( $Q$ is a first-order formula, $\alpha, \beta$ are hybrid programs)

\begin{tabular}{ll}
\hline \multicolumn{1}{c}{ Statement } & \multicolumn{1}{c}{ Effect } \\
\hline$\alpha ; \beta$ & sequential composition where $\beta$ starts after $\alpha$ finishes \\
$\alpha \cup \beta$ & nondeterministic choice, following either alternative $\alpha$ or $\beta$ \\
$\alpha^{*}$ & nondeterministic repetition, repeating $\alpha$ times for any $n \in \mathbb{N}$ \\
$x:=\theta$ & discrete assignment of the value of term $\theta$ to variable $x$ (jump) \\
$x:=*$ & nondeterministic assignment of an arbitrary real number to $x$ \\
$\left(x_{1}^{\prime}=\theta_{1}, \ldots\right.$, & continuous evolution of $x_{i}$ along the differential equation system \\
$\left.\quad x_{n}^{\prime}=\theta_{n} \& Q\right)$ & $x_{i}^{\prime}=\theta_{i}$ restricted to remain in evolution domain $Q$ at all times \\
$? Q$ & test if formula $Q$ holds at current state, abort program otherwise \\
if $(Q) \alpha$ & run $\alpha$ if $Q$ is true at current state, do nothing otherwise \\
if $(Q) \alpha$ else $\beta$ & run $\alpha$ if $Q$ is true at current state, run $\beta$ otherwise \\
\hline
\end{tabular}

Table 2: Operators of differential dynamic logic $(\mathrm{d} \mathcal{L})$ formulas

\begin{tabular}{lll}
\hline $\mathrm{d} \mathcal{L}$ & Operator & Meaning \\
\hline$\theta_{1} \sim \theta_{2}$ & comparison & true iff $\theta_{1} \sim \theta_{2}$ with operator $\sim \in\{>, \geq,=, \neq, \leq,<\}$ \\
$\neg \phi$ & negation / not & true if $\phi$ is false \\
$\phi \wedge \psi$ & conjunction / and & true if both $\phi$ and $\psi$ are true \\
$\phi \vee \psi$ & disjunction / or & true if $\phi$ is true or if $\psi$ is true \\
$\phi \rightarrow \psi$ & implication / implies & true if $\phi$ is false or $\psi$ is true \\
$\phi \leftrightarrow \psi$ & bi-implication / equivalent & true if $\phi$ and $\psi$ are both true or both false \\
$\forall x \phi$ & universal quantifier & true if $\phi$ is true for all values of variable $x$ in $\mathbb{R}$ \\
$\exists x \phi$ & existential quantifier & true if $\phi$ is true for some values of variable $x$ in $\mathbb{R}$ \\
{$[\alpha] \phi$} & {$[\cdot]$ modality / box } & true if $\phi$ is true after all runs of hybrid program $\alpha$ \\
$\langle\alpha\rangle \phi$ & $\langle\cdot\rangle$ modality / diamond & true if $\phi$ is true after at least one run of $\alpha$ \\
\hline
\end{tabular}

Note that the operator precedence is such that unary operators bind stronger than binary operators and, just like in regular expressions, ; binds stronger than $\cup$. In particular, the controller in (3) is

$$
(?(v \leq 5) ; a:=A) \cup a:=-b
$$

ASCII syntax. The benchmark examples are specified in the $\mathrm{d} \mathcal{L}$ ASCII syntax and grouped into . kyx files, each containing several named archive entries. The ASCII syntax is a straightforward ASCII rendition of Tables 1 and 2, e.g., using A->B for $A \rightarrow B$ and using A\&B for $A \wedge B$. The ASCII notation alpha++beta is used for alpha $\cup$ beta. For improved readability in longer examples, braces $\{\ldots\}$ are used for grouping differential equation systems and other program operators. Like in C programs, assignments etc. end with explicit semicolons.

Archive entries follow the general shape below, listing optional definitions, system variables, a (safety) specification in $\mathrm{d} \mathcal{L}$, and optional tactic scripts. The example (3), specialized, just for the sake of illustration, to the case where $A=5$, is written in ASCII KeYmaera X input as follows. Unlike the ProgramVariables and Problem block, the Definitions and Tactic blocks are optional. The symbols defined in the Definitions can be used in the Problem block or in other definitions. 
ArchiveEntry "Benchmark Example 1".

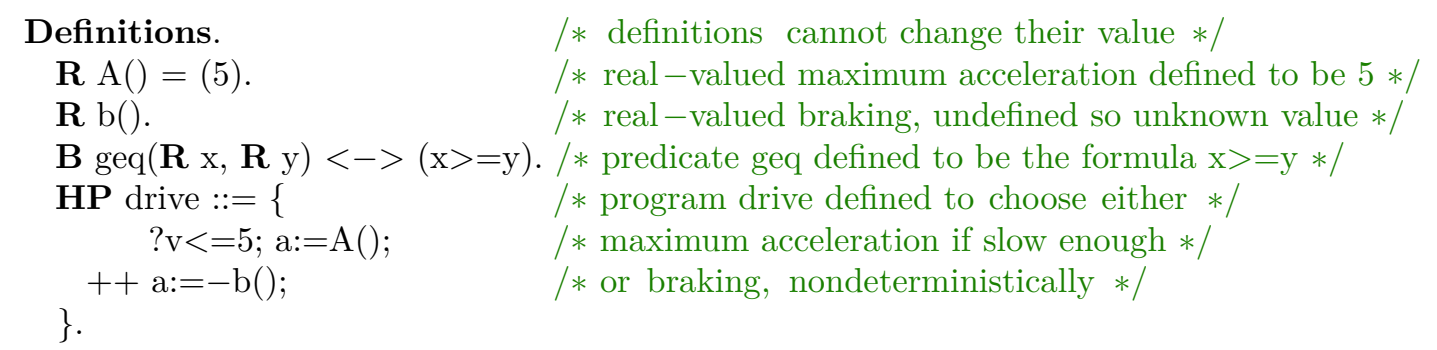

\section{End.}

ProgramVariables. / $*$ program variables may change their value over time $*$ /

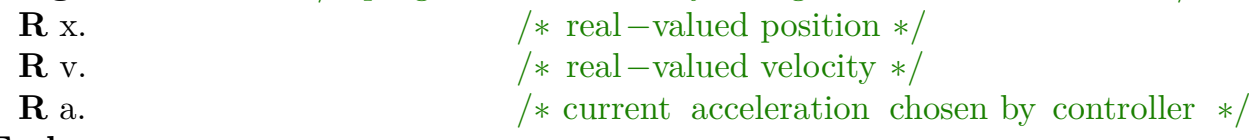

End.

Problem.

$\mathrm{v}>=0 \& \mathrm{~A}()>0 \& \mathrm{~b}()>0$

$->$

[

\{

drive;

/* conjecture in differential dynamic logic */

$\left\{\mathrm{x}^{\prime}=\mathrm{v}, \mathrm{v}^{\prime}=\mathrm{a} \& \mathrm{v}>=0\right\}$

$/ *$ initial condition $* /$

/* implies $* /$

/* all runs of this hybrid program $* /$

/* braces \{\} group programs $* /$

\}* @invariant $(\mathrm{v}>=0)$

] $\mathrm{v}>=0$

/* expand program drive here as defined above $*$ /

/* differential equation system $*$ /

/* loop repeats, with @invariant contract */

End.

/* safety/postcondition after hybrid program */

Tactic "Automated proof in KeYmaera X".

master

End.

Tactic "Scripted proof in Bellerophon tactic language".

$\operatorname{implyR}(1) ; \operatorname{loop}\left(\left\{{ }^{6} \mathrm{v}>=0^{`}\right\}, 1\right) ;<(\quad / *<$ splits separate branches $* /$

closeId, $\quad / *$ initial case: shown with close by identity $* /$

QE, $\quad / *$ postcondition: prove by real arithmetic $\mathrm{QE} * /$

/* induction step: decomposes hybrid program semi-explicitly $* /$

composeb(1) ; solve(1.1) ; $\operatorname{choiceb(1)~;~andR(1)~;~}<($ /* controller branches $* /$

composeb(1) ; testb(1) ; master, / / decompose some steps then ask master $* /$

$\operatorname{assignb}(1) ; \mathrm{QE} \quad / *$ assignment, then real arithmetic $* /$ )

)

End.

End. / $*$ end of ArchiveEntry */ 
Background. A short survey on differential dynamic logic and hybrid programs can be found in a LICS'12 tutorial [Pla12a], a tutorial on its modeling principles in STTT [QML ${ }^{+}$16], a research monograph [Pla10b], and a comprehensive introduction in a textbook [Pla18]. The precise mathematical semantics of differential dynamic logic and its hybrid programs can be found in the literature as well, for example the most recent details in [Pla17], and a brief version in the LICS'12 tutorial [Pla12a].

\section{Participating Tools}

KeYmaera $\mathbf{X}$. KeYmaera $\mathrm{X}\left[\mathrm{FMQ}^{+} 15\right]$ is a theorem prover for the hybrid systems logic differential dynamic logic $(\mathrm{d} \mathcal{L})$. It implements the uniform substitution calculus of $\mathrm{d} \mathcal{L}$ [Pla17]. ${ }^{1}$ KeYmaera X supports systems with nondeterministic discrete jumps, nonlinear differential equations, nondeterministic input, and it provides invariant construction and proving techniques for differential equations [SGJP16, PT18]. Unlike numerical hybrid systems reachability analysis tools, KeYmaera X also supports unbounded initial sets and unbounded time analysis.

KeYmaera X comes with automated proof search procedures that can be steered in the following ways: annotations in the input models provide additional design insight and, if available, are used to steer the invariant generation techniques in KeYmaera X; fine-grained control over proofs is available with proof scripts [FMBP17].

Extension with and experimentation in proof search without reducing trust in the prover is made possible on top of a small trusted kernel that checks all reasoning steps for soundness. The prover kernel contains a list of sound $\mathrm{d} \mathcal{L}$ axioms that are instantiated using a uniform substitution proof rule [Pla17]. This approach isolates all soundness-critical reasoning in the prover kernel and obviates the intractable task of ensuring that each new proof search algorithm is implemented correctly. New proof search algorithms are always sound and can either be programmed directly in Scala (or Java) or can simply be added as a tactic in the hybrid systems tactic language Bellerophon [FMBP17].

The proof automation for differential equations makes use of insights on how to prove all invariants of differential equations [PT18]. Tactical implementations allow KeYmaera X to soundly reduce ODE invariance questions to a small number of core ODE axioms and real arithmetic. The proof tactic is optimized for fast proofs of commonly used invariants, e.g., barrier certificates [PJP07]. All real arithmetic questions that arise in the proofs are rigorously checked, including the ones that arise from the use of barrier certificates. This guarantees that any barrier certificate that proves with KeYmaera $\mathrm{X}$ are true barrier certificates, rather than the result of numerical or floating-point errors.

To prove properties of differential equations, KeYmaera X combines an axiomatic differential equation solver [Pla17] and local fixedpoint computation for differential invariants [PC09a] with tactics based on differential equation axiomatization [PT18] and Pegasus, a toolbox for automatically generating continuous invariants for systems of ordinary differential equations. Given a system of ODEs subject to an evolution domain constraint, a set of initial states, and a set of unsafe states, Pegasus will attempt to automatically generate a continuous invariant that is sufficient to prove that the ODE cannot continuously evolve into an unsafe state from any of its initial states while respecting the evolution constraint. Pegasus is implemented in Mathematica and at present relies on an array of techniques from qualitative analysis and discrete abstraction [SGJP16] for constructing continuous invariants.

${ }^{1}$ This $\mathrm{d} \mathcal{L}$ uniform substitution calculus is also formally verified in Isabelle/HOL and $\left.\mathrm{Coq}^{\left[\mathrm{BRV}^{+}\right.} 17\right]$. 
KeYmaera 3. KeYmaera 3 [PQ08] is the previous generation theorem prover for differential dynamic logic $\mathrm{d} \mathcal{L}$. Unlike its successor KeYmaera X, the older KeYmaera 3 directly implements a sequent calculus for differential dynamic logic [Pla08], instead of a uniform substitution calculus. What KeYmaera $\mathrm{X}$ implements from a few simple modular axioms, KeYmaera 3 uses several dedicated proof rules for [Pla08, Pla10a, Pla12b]. This leads to a more directly usable but substantially bigger soundness-critical prover kernel of about 66000 lines of code written in a mix of Java and Scala. In some cases, one single proof rule use, e.g., for solving differential equations in KeYmaera 3 corresponds to thousands of axiom uses in KeYmaera X. The impact on soundness, however, is that the ODE solver of KeYmaera 3 is trusted while that of KeYmaera $\mathrm{X}$ is not trusted, because each of its outputs is verified with a proof.

For proof automation, KeYmaera 3 implements a simple but fast fixpoint loop [PC09a] for generating loop invariants of hybrid systems and differential invariants of differential equations. It provides an array of different SMT strategies for splitting real arithmetic subquestions [Pla10b]. Changing proof search procedures in KeYmaera 3 (beyond choosing from the list of predefined ones) is significantly more complicated and, notably, soundness-critical.

HHL Prover. HHL Prover [WZZ15] is an interactive theorem prover implemented in Isabelle/HOL [NPW02] to mechanize the Hybrid Hoare Logic (HHL) deductive calculus [LLQ ${ }^{+}$10] for verifying hybrid systems modeled by the Hybrid CSP (HCSP) [He94, ZWR96].

HCSP [He94, ZWR96] is an extension of CSP by introducing differential equations for representing continuous evolution and several forms of interruptions to continuous evolution. For a sequential HCSP process $P$, the specification takes the form $\{$ Pre $\} P\{$ Post; HF $\}$, where the pre-/post-condition Pre and Post, defined by first-order logic, specify properties of variables that hold at the beginning and termination of the execution of $P$ respectively, and the history formula $H F$, defined by duration calculus [ZHR91, CH04], specifies properties of variables that hold throughout the execution interval of $P$. The specification for a parallel process $P_{1} \| P_{2}$ is then defined by assigning to each sequential component of it the respective pre-/post-conditions and the history formula, shown as below:

$$
\left\{\text { Pre }_{1}, \text { Pre }_{2}\right\} P_{1} \| P_{2}\left\{\text { Post }_{1}, \text { Post }_{2} ; H F_{1}, H F_{2}\right\}
$$

HHL axiomatizes HCSP constructs by a set of axioms and inference rules, which constitutes a basis for implementing the verification condition generator for verifying HCSP specifications in HHL prover.

The implementation of HHL prover can be found at [WZZ15]. The proof in HHL prover is performed according to the following process: first, by applying HHL rules, a HCSP specification is transformed step by step to a set of high-order logic (HOL) formulas, i.e. verification conditions; and then, by applying proof tactics and rules of HOL, the validity of verification conditions, that is equivalent to the correctness of the original HCSP specification, is proved. However, when the specification to be proved contains unknown differential invariants [LZZ11, PC08], some verification conditions related to the invariants remain unproved in HHL Prover. For such cases, the prover needs to call external invariant generators for solving the invariants.

HHL Prover has been integrated into a tool chain called $M A R S\left[\mathrm{CHT}^{+} 17\right]$ for Modeling, Analyzing and veRifying hybrid Systems. Using MARS, firstly, executable models of hybrid systems are built with the industrial standard environment Simulink/Stateflow, and then Simulink/Stateflow diagrams are translated into HCSP processes by an automatic translator Sim2HCSP [ZZWF15], and finally the HCSP processes can be verified preserving the given properties using the HHL Prover. MARS is later extended with a code generator that 
translates the verified HCSP with continuous behavior to discrete SystemC code [YJL $\left.{ }^{+} 16\right]$. It is guaranteed that the source HCSP model and the target SystemC code are approximately bisimilar.

\section{Benchmarks}

One of the strengths of hybrid systems theorem proving as a verification technique is its support for combined automated and interactive verification steps as well as its applicability to proof search and proof checking. The benchmark examples were analyzed in three modes:

Automated The specification is the only input to the theorem prover. Proofs and counterexamples are obtained fully automated to highlight the capabilities of theorem provers in terms of invariant generation, proof search, and proof checking.

Hints Known design properties of the system, such as loop invariants and invariants of differential equations, are annotated in the model and allowed to be exploited during an otherwise fully automated proof to highlight the capabilities of theorem provers in terms of proof search and proof checking.

Scripted User guidance with proof scripts is allowed to highlight the capabilities of theorem provers in terms of proof checking.

The benchmark examples are structured into 3 categories: hybrid systems design shape examples to test for system design variations at a small scale, nonlinear continuous models to test for continuous invariant construction and proving capabilities, and hybrid systems case studies to test for prover scalability.

Experimental setup. The machines used to run the benchmark examples are listed in Appendix A: KeYmaera X (in automated (A), hints (H), and scripted (S) mode) and KeYmaera 3 (in automated (A) mode) participated on all benchmark sets and were executed on the same machine $\mathrm{M}_{\mathrm{k}}$, and therefore their computation times are directly comparable. HHL Prover participated with the Chinese Train Control System case study on its own machine $\mathrm{M}_{\mathrm{hhl}}$. The execution time measurements were taken separately on a fresh prover instance for each example in the benchmark set. Proof attempts were aborted after a category-specific timeout, well above the longest successful solution in the category. The competition results are presented with accumulated execution times after examples are ranked according to their execution time.

\subsection{Hybrid Systems Design Shapes}

Category overview. In this category, basic examples ${ }^{2}$ test for proof automation techniques for a large variety of system designs: event-triggered systems, time-triggered systems, systems with nested loops and differential equations, and systems with model-predictive control. Instead of focusing on particularly complex systems, this set of examples strives at a certain degree of coverage of qualitatively different kinds of systems and their different typical shapes. The benchmark examples are grouped as follows:

Static semantics correctness 9 examples with various sequential orders and nested structures of assignments, differential equations, and loops.

Dynamics 30 examples with differential equations ranging from solvable to nonlinear.

\footnotetext{
${ }^{2}$ https://github.com/LS-Lab/KeYmaeraX-projects/blob/master/benchmarks/basic . kyx
} 


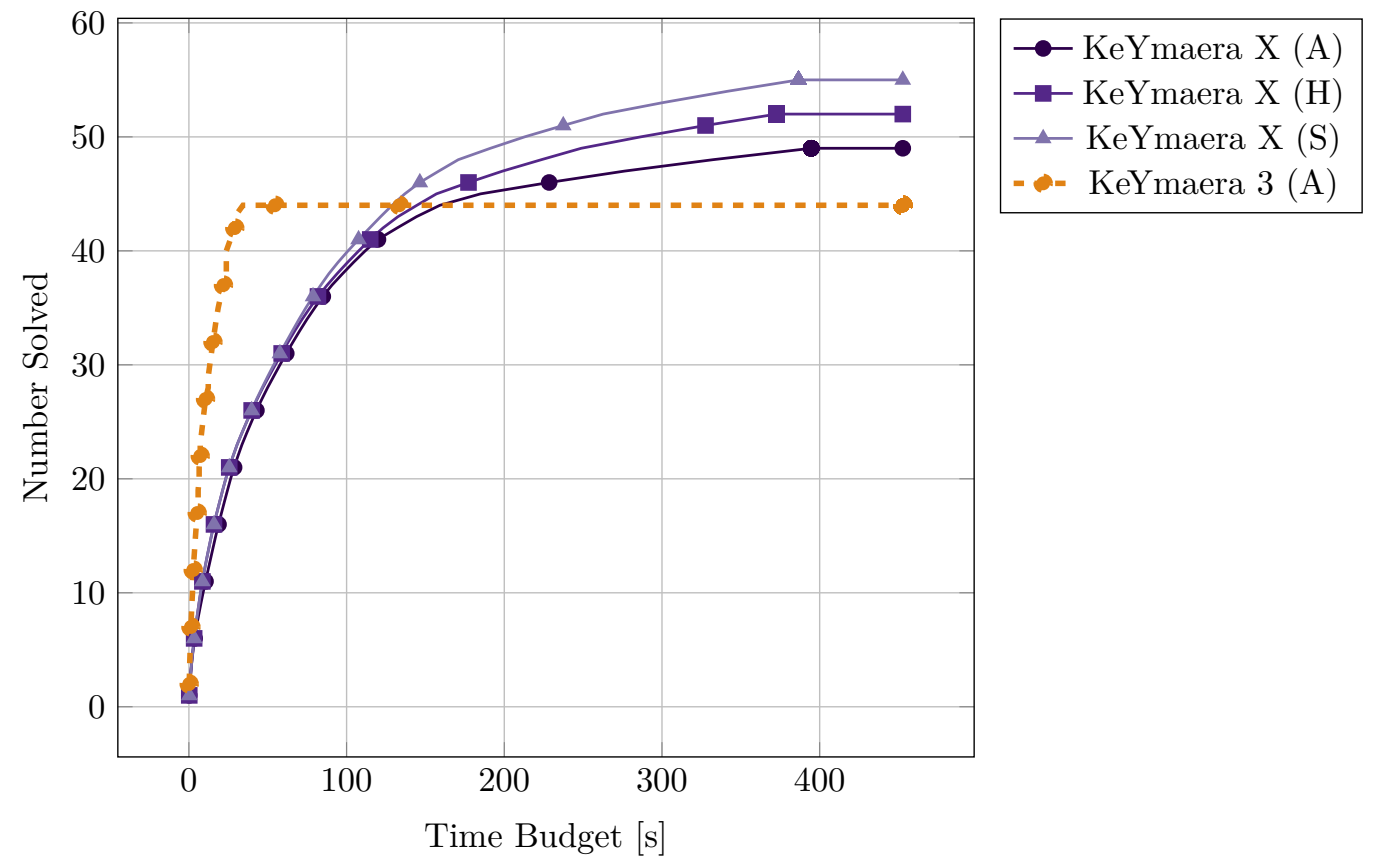

Figure 1: Computation times: Basic benchmark examples. Ranked accumulated time budgets $[\mathrm{s}]$, which are the number of examples solved within a total accumulated time budget

LICS Tutorial $9 \mathrm{~d} \mathcal{L}$ tutorial examples [Pla12a] ranging from basic time-triggered motion control to model-predictive control.

STTT Tutorial $12 \mathrm{~d} \mathcal{L}$ modeling tutorial examples [QML $\left.{ }^{+} 16\right]$ ranging from basic discrete event-triggered and time-triggered control for straight-line motion to speed control with a trajectory generator and lane-keeping with two-dimensional curved motion.

Competition results. Proof attempts were aborted after a timeout of $300 \mathrm{~s}$ in the basic category, with the longest successful solution after about $62 \mathrm{~s}$. The results for the basic category in terms of accumulated execution times are shown in Fig. 1. For example, the fastest 30 fully automated examples can all be solved in cumulative time $13.3 \mathrm{~s}$ in KeYmaera 3 vs. $57.7 \mathrm{~s}$ in KeYmaera X (note that the sets of fastest examples are not necessarily the same). The main insight from Fig. 1 is that automated proof search with a fixpoint loop in KeYmaera 3 is faster than the proof search in KeYmaera X for the solved examples, but KeYmaera X solves a larger portion of the benchmark set with the additional time it takes. Hints and proof scripts in KeYmaera X help speed up a little bit and solve additional examples. This indicates that the primary impact of further proof automation for the basic category of benchmarks will not be the resulting speed but the number of examples that can be proved fully automatically.

\subsection{Nonlinear Continuous Models}

Category overview. This set of 69 nonlinear continuous safety verification problems ${ }^{3}$ is based on the problems proposed in [SGJ16]. The problems in this benchmark set were gath-

\footnotetext{
${ }^{3}$ https://github.com/LS-Lab/KeYmaeraX-projects/blob/master/benchmarks/nonlinear.kyx
} 
ered from published papers in the area of continuous safety verification and invariant generation for nonlinear systems ([DGXZ17, LZZ11, DCKB17, SGS14, SGJP16]). The bulk of the problems in the benchmark set feature planar (i.e., 2-dimensional) polynomial systems of ODEs in which the safety property is known to hold for unbounded time. The ODEs are furthermore autonomous (i.e., do not explicitly depend on the independent time variable $t$ ); this fact presents no real restriction since non-autonomous ODEs can be brought into autonomous form by augmenting the dynamics with $t^{\prime}=1$. Certain non-polynomial systems of ODEs can likewise be brought into polynomial form by introducing fresh variables in a process called re-casting [SV87]. While we stress that the existing set of nonlinear polynomial ODE safety benchmarks can in no way be said to be representative (owing to its small size), the general class of problems which fits into this category is highly important.

Example 4.1. The nonlinear system from [DLA06, Ex. 5.2. ii] that was shown in (2) has the following dynamics:

$$
\begin{aligned}
x^{\prime} & =2 x-2 x y, \\
y^{\prime} & =2 y-x^{2}+y^{2} .
\end{aligned}
$$

Taking the initial states to be $-\frac{4}{5}<x<-\frac{1}{3} \wedge-1 \leq y<0$ and $(x=0 \wedge y=0) \vee x+y>1$ to be the forbidden states, the verification problem is illustrated in Fig. 2.

Competition results. Proof attempts in the nonlinear category were aborted after a timeout of $300 \mathrm{~s}$, well above the longest successful solution of about $42 \mathrm{~s}$ in automated mode and $51 \mathrm{~s}$ in scripted mode. Fig. 3 plots the accumulated execution times for the nonlinear category after examples are ranked according to their execution time. The main insight from Fig. 3 is that the invariant construction [SGJP16] and proving techniques [PT18]

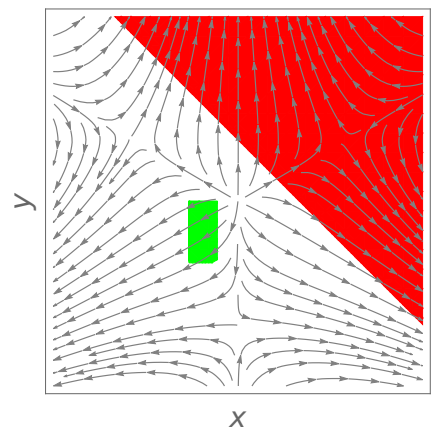

Figure 2: Nonlinear continuous safety verification problem. No initial state (green rectangle) can evolve into unsafe states (red halfplane) along the trajectories. of KeYmaera X significantly outperform and improve upon KeYmaera 3 the extent to which continuous dynamics can be analyzed fully automatically. Even proof hints have a negligible impact compared to full automation. ${ }^{4}$ The results in scripted mode (S) emphasize the generality of the implemented proving techniques for differential equations: proof scripts with barrier certificates that were generated outside KeYmaera X solve almost all the remaining examples. This highlights a potential to improve automated invariant construction with methods to construct barrier certificates, which are plagued by numerical robustness issues.

\subsection{Hybrid Systems Case Study Benchmarks}

Category overview. The benchmark examples in this category are selected to test theorem provers for scalability and efficiency on examples of a significant size and interest in applications. The benchmark examples ${ }^{5}$ are inspired from prior case studies on train control [PQ09, ZLW $\left.{ }^{+} 14\right]$, flight collision avoidance [PC09b], and robot collision avoidance [MGVP17].

\footnotetext{
${ }^{4}$ But this observation could be sensitive to the chosen benchmarks.

${ }^{5}$ https://github.com/LS-Lab/KeYmaeraX-projects/blob/master/benchmarks/advanced.kyx
} 


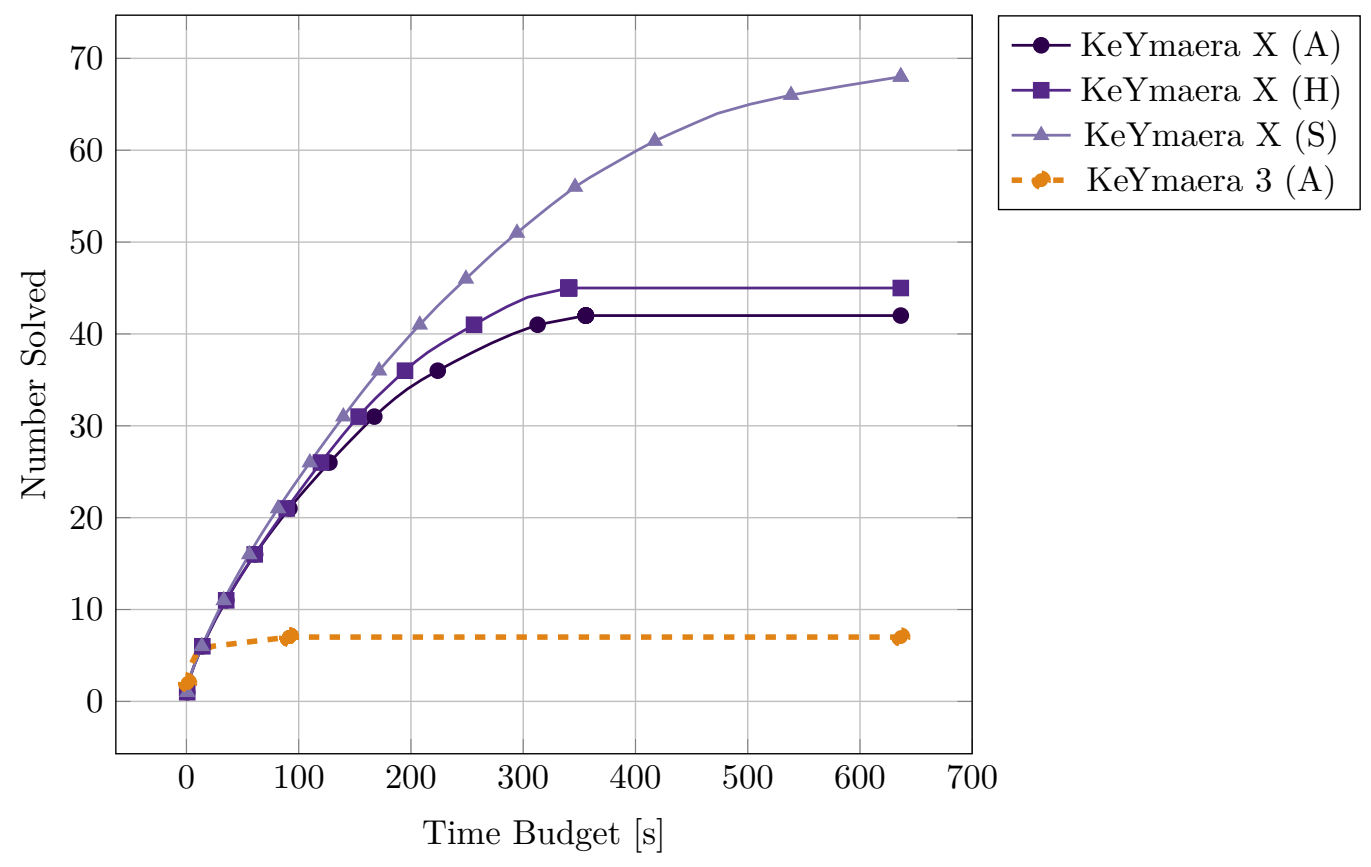

Figure 3: Computation times: Nonlinear benchmark examples. Ranked accumulated time budgets [s], which are the number of examples solved within a total accumulated time budget

European train control system (ETCS). This benchmark on automated train control bases on the safety analysis [PQ09] of the cooperation protocol in the European Train Control System [ERT02, DHO03], which specifies the interaction between an automated train protection system and a radio-block controller. The radio-block controller (purely discrete dynamics) may at any time issue speed limits that take effect at certain positions; the train must respect these speed limits (hybrid dynamics of train controller and train motion).

E-1 (ETCS: Essentials) Describes the core safety theorem: a time-triggered train controller never violates the posted speed limit.

E-2 (ETCS: Proposition 1 (Controllability)) Describes the motion of a train on brakes and translates it into a stopping distance. Tests a prover's ability to show equivalence between a hybrid systems specification in $\mathrm{d} \mathcal{L}$ and it's core information in terms of stopping distance in real arithmetic.

E-3 (ETCS: Proposition 4 (Reactivity)) Describes the motion of a train when accelerating for a bounded amount of time and the necessary distance to a full stop. Tests a prover's ability to work with universally quantified assumptions and/or analyze programs in the context of universally quantified input.

The benchmark tests a prover's ability to handle $\mathrm{d} \mathcal{L}$ safety properties (modal formulas) in various places of a specification, for example, as proof obligations and as assumptions.

Chinese train control systems (CTCS). This case study is about modeling and verification of a combined operational scenario of Chinese Train Control System Level-3 (CTCS-3). It originates from an under-specification error of the System Requirements Specification (SRS) of CTCS-3, revealed during a spot testing of the system, which caused a train to stop unexpect- 


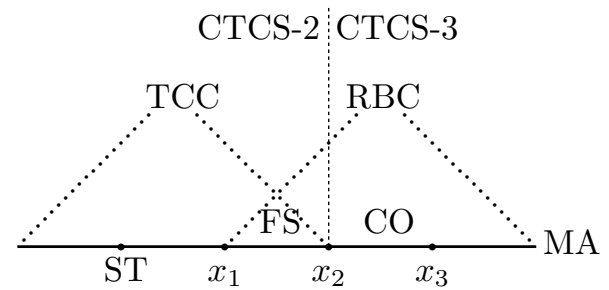

Figure 4: A combined scenario of CTCS-3.

edly. It has been studied in $\left[\mathrm{ZLW}^{+} 14, \mathrm{ZZW}^{+} 13, \mathrm{ZZWF} 15\right]$ and the failure was reproduced by simulation and also formally verified.

The combined scenario integrates the movement authority (MA) scenario, the level transition (from CTCS-2 to CTCS-3) scenario, as well as the mode transition (from Full Supervision mode to Calling On mode, FS to CO for short) scenario of CTCS-3. The combined scenario is shown in Fig. 4, which occurs under the following situation:

- The train has got enough MA to complete the combined scenario, and

- There are two adjacent segments in the MA, divided by location $x_{2}$. At $x_{2}$, the level transition from CTCS-2 to CTCS-3, and the mode transition from FS to CO, will occur simultaneously, and

- The train starts to move at location $S T$, and has an agreement from RBC (Radio Block Center) to start level transition at $x_{1}$ and complete the level transition at $x_{2}$.

According to the SRS, the combined scenario is required to satisfy a liveness property: the train can eventually move beyond the location $x_{2}$ with a positive speed, with both the level transition and mode transition completed successfully.

However, the under-specified SRS fails to guarantee the liveness property. Basically, for safety reasons, to switch from FS mode to CO mode under CTCS-3, the driver's confirmation is required before the switching point $x_{2}$ to upgrade the speed limit of the CO mode, which is originally set to 0 . However, in the old version of the SRS, such a confirmation request is not explicitly specified to be issued to the driver during a region where the train is co-supervised by both CTCS-2 and CTCS-3 ( $x_{1}$ to $x_{2}$ in Fig. 4 ). As a result, the speed limit of the CO segment cannot be upgraded and remains 0 , which forces the train to stop at $x_{2}$. Thus the verification objective for this case study is to prove on the underspecified model the negation of the liveness property, that is, the train must stop at $x_{2}$.

Roundabout air traffic conflict resolution (ATC). Air traffic conflict resolution maneuvers with curved flight dynamics exhibit nontrivial interactions of discrete and continuous dynamics. The roundabout benchmark [PC09a] is based on [TPL ${ }^{+}$96, TPS98, HHMW00, MF01, DPR05, PC09b, PKV09] to analyze collision freedom of planar roundabout maneuvers in air traffic control that should guarantee safe spatial separation of aircraft throughout their flight. The scale of this benchmark can be adjusted easily with the number of aircraft involved in the conflict resolution maneuver: additional aircraft increase the number of variables in the benchmark and introduce additional invariants that must be found, but analysis is separable into pairwise collision freedom questions.

A-2 (ATC: 2 Aircraft Tangential Roundabout Maneuver) Describes the circular conflict resolution of two aircraft in a planar roundabout collision avoidance maneuver.

A-3 (ATC: 3 Aircraft Tangential Roundabout Maneuver) Circular conflict resolution of three aircraft in planar roundabout collision avoidance maneuvers. Safety of the entire 
system is collision-freedom between all three aircraft pairs.

A-4 (ATC: 4 Aircraft Tangential Roundabout Maneuver) Circular conflict resolution of four aircraft in planar roundabout collision avoidance maneuvers. Safety of the entire system is collision-freedom between all six aircraft pairs.

The benchmark tests a prover's ability to analyze nested loops and multiple nonlinear differential equations. At larger numbers of aircraft it also tests the scale of reasoning about nonlinear dynamics by identifying and splitting analysis into isolated sub-questions.

Robot collision avoidance (RX). This benchmark bases on [MGVP17] and analyzes obstacle avoidance in ground robot navigation. The benchmark uses models and safety properties to analyze collision avoidance safety in the presence of stationary obstacles and moving obstacles.

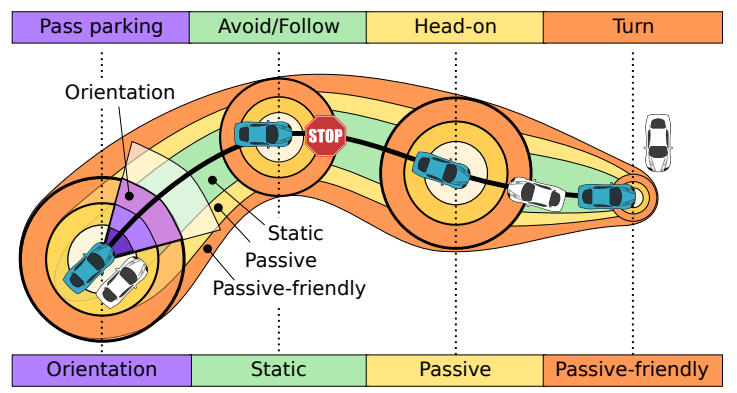

Figure 5: Robot collision avoidance properties: benchmark tests static safety and passive safety.

The resulting real arithmetic formulas describing the Euclidian distance between robot and obstacle after symbolic execution are challenging for current solvers and may require overapproximation and simplification in the theorem prover steering the backend decision procedures.

R-1 (Robot collision avoidance: static safety) ensures that no collisions can happen with stationary obstacles. Tests a prover's ability to handle mixed solvable (longitudinal robot acceleration) and nonlinear (rotational robot motion) continuous dynamics, and its ability to overapproximate norms (Euclidian distance overapproximated to infinity norm).

R-2 (Robot collision avoidance: passive safety) ensures that no collisions can happen with stationary or moving obstacles while the robot moves. The size of the resulting real arithmetic formulas are challenging for current solvers even after overapproximation of Euclidian distances. Tests a prover's ability to steer backend decision procedures by selecting relevant assumptions, using monotonicity arguments to eliminate variables, and simplify arithmetic.

This benchmark tests a prover's ability to analyze mixed solvable and nonlinear differential equations, overapproximation of norms, and arithmetic simplifications.

Competition results. Proof attempts in the hybrid systems case study category were aborted after a timeout of $1500 \mathrm{~s}$, with the longest successful proof after about $938 \mathrm{~s}$. Table 3 lists the individual computation times for each of the case study benchmark examples, Fig. 6 summarizes the accumulated computation times. KeYmaera 3 and KeYmaera X participated on the full benchmark set (CTCS attempted only in automated (A) mode, future verification with hints and scripts is planned), whereas HHL Prover participated only on the CTCS case study. Again, 


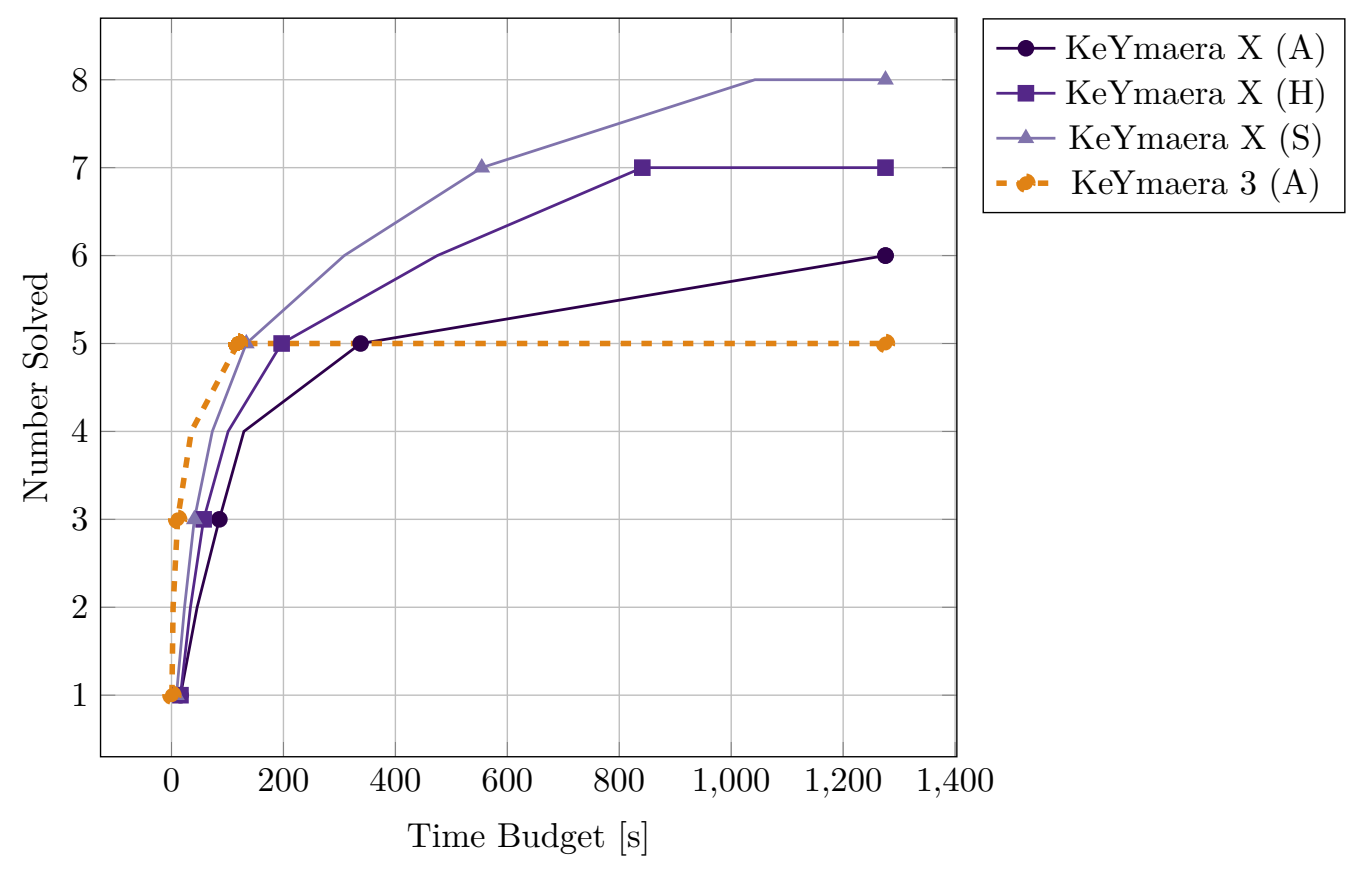

Figure 6: Computation times: Case study benchmark examples. Ranked accumulated time budgets [s], which are the number of examples solved within a total accumulated time budget

the fixpoint loop invariant generation technique in KeYmaera 3 solves examples fast, while hints and proof scripts in KeYmaera $\mathrm{X}$ help scale. The results point out a potential to improve tactic implementation efficiency, since on the hybrid systems case studies that KeYmaera 3 can solve automatically it outperforms proof checking from hints in KeYmaera X. The ETCS benchmark examples feature solvable continuous dynamics, which unsurprisingly leads to a significant computation time difference between the ODE solution sequent rule in KeYmaera 3 and the proof-producing tactic in KeYmaera X. The ATC benchmark examples highlight a particularly useful proof scalability technique in KeYmaera 3, which splits conjunctive safety properties into separate proof obligations. This technique is mimicked in the KeYmaera $\mathrm{X}$ scripted mode to reduce computation time, for example, in ATC A-4 from $937.8 \mathrm{~s}$ to $245.7 \mathrm{~s}$, but requires further tactic improvements to get to the computational efficiency of KeYmaera 3. The robot colli-

Table 3: Computation times $[\mathrm{s}]$

\begin{tabular}{|c|c|c|c|c|c|c|c|c|c|c|}
\hline \multirow{2}{*}{ Tool } & & \multicolumn{3}{|c|}{ ETCS } & \multirow[b]{2}{*}{ CTCS-3 } & \multicolumn{3}{|c|}{ ATC } & \multicolumn{2}{|c|}{$\mathrm{RX}$} \\
\hline & & E-1 & E-2 & E-3 & & A-2 & A-3 & A-4 & $\mathrm{R}-1$ & $\mathrm{R}-2$ \\
\hline \multirow{3}{*}{ KeYmaera X } & (A) & 29.7 & 16.1 & 43.8 & - & 39.7 & 208.5 & 937.8 & - & - \\
\hline & (H) & 23.6 & 16.0 & 43.7 & - & 17.8 & 95.6 & 366.3 & 278.2 & - \\
\hline & (S) & 17.6 & 14.4 & 32.1 & - & 8.8 & 60.8 & 245.7 & 175.0 & 488.0 \\
\hline KeYmaera 3 & (A) & 2.1 & 0.7 & - & - & 8.1 & 24.3 & 83.4 & - & - \\
\hline HHL Prover & $(\mathrm{S})$ & - & - & - & 59 & - & - & - & - & - \\
\hline
\end{tabular}




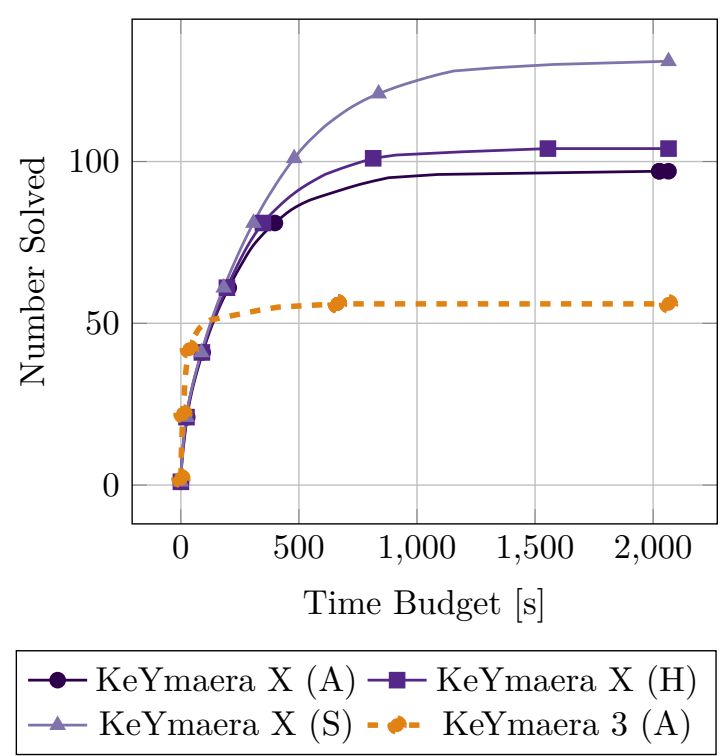

(a) Ranked accumulated time budgets [s]: number of examples solved in total accumulated time budget

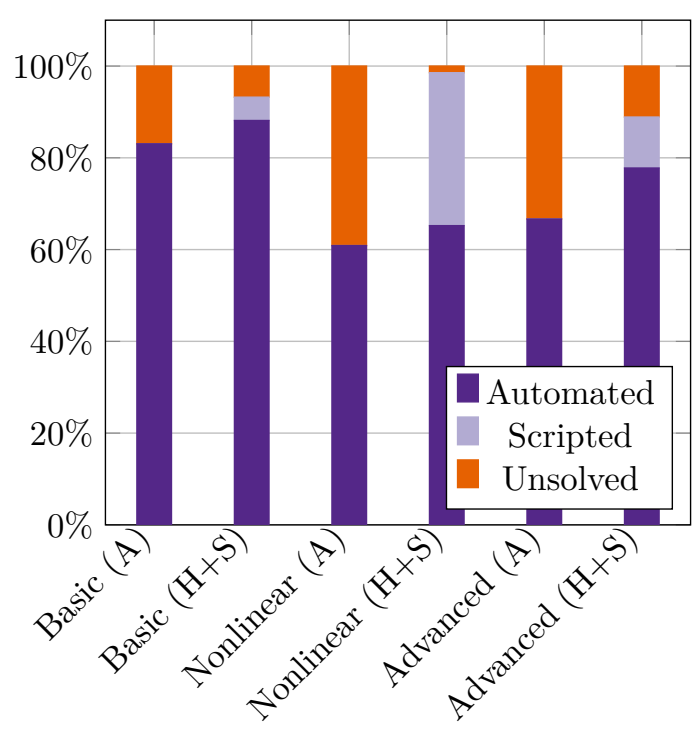

(b) KeYmaera X: Number solved automated $(\mathrm{A})$, hints $(\mathrm{H})$, and scripted $(\mathrm{S})$

Figure 7: Result summary: KeYmaera 3 is faster but solves less examples, especially among those with nonlinear dynamics. KeYmaera X scales better; hints and scripts increase the number of solved examples and reduce computation time.

sion avoidance benchmark examples illustrate where current automation fails to find invariants and identify the necessary arithmetic simplifications for backend procedures to complete in reasonable time; proof checking from hints and scripts for arithmetic simplifications illustrate potential ways forward to improve proof search automation.

Note HHL Prover. A Simulink/Stateflow model has been built for the combined scenario in the CTCS-3 case study. Applying the tool Sim2HCSP to the Simulink/Stateflow model, seven files were generated which describe the HCSP model as well as the goal to be verified. Then using HHL Prover, the goal was proved successfully as a theorem, taking 59 seconds to finish on the $\mathrm{M}_{\mathrm{hhl}}$ platform with Intel Core i7-4790 CPU 3.60GHZ and 16GB memory. In particular, during the interactive proof process, certain differential invariants were manually fed into the HHL specification.

\section{Conclusion and Outlook}

The hybrid systems theorem proving friendly competition focuses on the characteristic features of hybrid systems theorem proving: flexibility of programming language principles for hybrid systems, unambiguous program semantics, and mathematically rigorous logical reasoning principles.

The (almost 140) benchmark examples are chosen to reflect a large variety of hybrid systems model shapes and scales to test hybrid systems theorem provers both for their flexibility to 


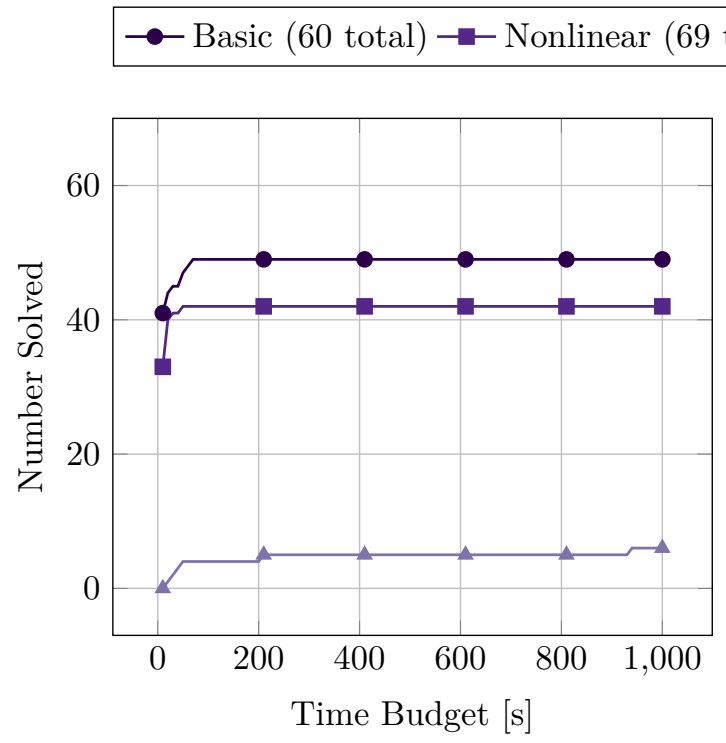

(a) KeYmaera X

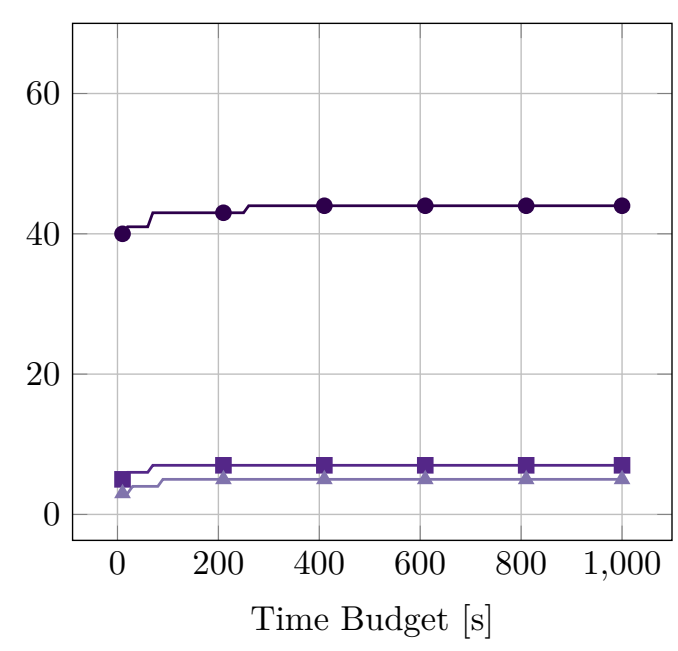

(b) KeYmaera 3

Figure 8: Result summary: Number of examples solvable fully automatically (A) with individual time budgets. KeYmaera X solves more examples, especially among those with nonlinear dynamics.

analyze typical modeling styles and for their scalability. More potential benchmark examples are always welcome in future years of the competition! The hybrid systems theorem proving category allows tools to choose their operating mode on the spectrum from fast proof checking of scripted proofs, hint-supported proof search and checking, to full automation.

The results, summarized in Figures 7 and 8, show significant improvements over theorem prover generations (KeYmaera X compared to its predecessor KeYmaera 3) in handling continuous dynamics fully automatically, but also highlight that the tactics in KeYmaera X can still learn in terms of performance from the proof search and checking procedures of KeYmaera 3.

Acknowledgments. This material is based upon work supported by the National Science Foundation under CNS-1739629 and by the AFOSR under grant number FA9550-16-1-0288. Yong Kiam Tan was also supported by A*STAR, Singapore. Hengjun Zhao was supported by NSFC grant 61702425. Xiangyu Jin, Shuling Wang, and Naijun Zhan are funded partly by NSFC under grant No. 61625206 and 61732001, by "973 Program" grant No. 2014CB340701 and by the CAS/SAFEA International Partnership Program for Creative Research Teams.

\section{References}

$\left[\mathrm{BRV}^{+} 17\right]$ Brandon Bohrer, Vincent Rahli, Ivana Vukotic, Marcus Völp, and André Platzer. Formally verified differential dynamic logic. In Yves Bertot and Viktor Vafeiadis, editors, Certified Programs and Proofs - 6th ACM SIGPLAN Conference, CPP 2017, Paris, France, January 16-17, 2017, pages 208-221, New York, 2017. ACM. 
[CH04] Zhou Chaochen and Michael R. Hansen. Duration Calculus - A Formal Approach to Real-Time Systems. Monographs in Theoretical Computer Science. An EATCS Series. Springer, 2004.

$\left[\mathrm{CHT}^{+} 17\right]$ Mingshuai Chen, Xiao Han, Tao Tang, Shuling Wang, Mengfei Yang, Naijun Zhan, Hengjun Zhao, and Liang Zou. MARS: A Toolchain for Modelling, Analysis and Verification of Hybrid Systems, pages 39-58. Springer, 2017.

[DCKB17] Adel Djaballah, Alexandre Chapoutot, Michel Kieffer, and Olivier Bouissou. Construction of parametric barrier functions for dynamical systems using interval analysis. Automatica, 78:287-296, 2017.

[DGXZ17] Liyun Dai, Ting Gan, Bican Xia, and Naijun Zhan. Barrier certificates revisited. J. Symb. Comput., 80:62-86, May 2017.

[DHO03] Werner Damm, Hardi Hungar, and Ernst-Rüdiger Olderog. On the verification of cooperating traffic agents. In Frank S. de Boer, Marcello M. Bonsangue, Susanne Graf, and Willem P. de Roever, editors, FMCO, volume 3188 of LNCS, pages 77-110. Springer, 2003.

[DLA06] Freddy Dumortier, Jaume Llibre, and Joan C Artés. Qualitative Theory of Planar Differential Systems. Springer, 2006.

[DPR05] Werner Damm, Guilherme Pinto, and Stefan Ratschan. Guaranteed termination in the verification of LTL properties of non-linear robust discrete time hybrid systems. In Doron A. Peled and Yih-Kuen Tsay, editors, ATVA, volume 3707 of LNCS, pages 99-113. Springer, 2005.

[ERT02] ERTMS User Group. UNISIG: ERTMS/ETCS system requirements specification. http://www.era.europa.eu, 2002. Version 2.2.2.

[FMBP17] Nathan Fulton, Stefan Mitsch, Brandon Bohrer, and André Platzer. Bellerophon: Tactical theorem proving for hybrid systems. In Mauricio Ayala-Rincón and César A. Muñoz, editors, ITP, volume 10499 of LNCS, pages 207-224. Springer, 2017.

$\left[\mathrm{FMQ}^{+}{ }^{15}\right]$ Nathan Fulton, Stefan Mitsch, Jan-David Quesel, Marcus Völp, and André Platzer. KeYmaera X: An axiomatic tactical theorem prover for hybrid systems. In Amy Felty and Aart Middeldorp, editors, CADE, volume 9195 of $L N C S$, pages 527-538, Berlin, 2015. Springer.

[He94] Jifeng He. From CSP to hybrid systems. In A Classical Mind, Essays in Honour of C.A.R. Hoare, pages 171-189. Prentice Hall International (UK) Ltd., 1994.

[HHMW00] Thomas A. Henzinger, Benjamin Horowitz, Rupak Majumdar, and Howard Wong-Toi. Beyond HYTECH: hybrid systems analysis using interval numerical methods. In Nancy A. Lynch and Bruce H. Krogh, editors, HSCC, volume 1790 of LNCS, pages 130-144. Springer, 2000 .

$\left[\mathrm{LLQ}^{+} 10\right] \quad$ Jiang Liu, Jidong Lv, Zhao Quan, Naijun Zhan, Hengjun Zhao, Chaochen Zhou, and Liang Zou. A calculus for hybrid CSP. In Kazunori Ueda, editor, APLAS, volume 6461 of $L N C S$, pages 1-15. Springer, 2010.

[LZZ11] Jiang Liu, Naijun Zhan, and Hengjun Zhao. Computing semi-algebraic invariants for polynomial dynamical systems. In Samarjit Chakraborty, Ahmed Jerraya, Sanjoy K. Baruah, and Sebastian Fischmeister, editors, EMSOFT, pages 97-106. ACM, 2011.

[MF01] Mieke Massink and Nicoletta De Francesco. Modelling free flight with collision avoidance. In ICECCS, pages 270-280. IEEE Computer Society, 2001.

[MGVP17] Stefan Mitsch, Khalil Ghorbal, David Vogelbacher, and André Platzer. Formal verification of obstacle avoidance and navigation of ground robots. I. J. Robotics Res., 36(12):1312$1340,2017$.

[NPW02] Tobias Nipkow, Lawrence C. Paulson, and Markus Wenzel. Isabelle/HOL: A Proof Assistant for Higher-Order Logic, volume 2283 of LNCS. Springer, 2002.

[PC08] André Platzer and Edmund M. Clarke. Computing differential invariants of hybrid systems as fixedpoints. In Aarti Gupta and Sharad Malik, editors, CAV, volume 5123 of LNCS, pages 176-189. Springer, 2008. 
[PC09a] André Platzer and Edmund M. Clarke. Computing differential invariants of hybrid systems as fixedpoints. Form. Methods Syst. Des., 35(1):98-120, 2009. Special issue for selected papers from CAV'08.

[PC09b] André Platzer and Edmund M. Clarke. Formal verification of curved flight collision avoidance maneuvers: A case study. In Ana Cavalcanti and Dennis Dams, editors, FM, volume 5850 of $L N C S$, pages 547-562, Berlin, 2009. Springer.

[PJP07] Stephen Prajna, Ali Jadbabaie, and George J. Pappas. A framework for worst-case and stochastic safety verification using barrier certificates. IEEE T. Automat. Contr., 52(8):1415-1429, 2007.

[PKV09] Erion Plaku, Lydia E. Kavraki, and Moshe Y. Vardi. Hybrid systems: from verification to falsification by combining motion planning and discrete search. Form. Methods Syst. Des., 34(2):157-182, 2009.

[Pla08] André Platzer. Differential dynamic logic for hybrid systems. J. Autom. Reas., 41(2):143189, 2008.

[Pla10a] André Platzer. Differential-algebraic dynamic logic for differential-algebraic programs. J. Log. Comput., 20(1):309-352, 2010.

[Pla10b] André Platzer. Logical Analysis of Hybrid Systems: Proving Theorems for Complex Dynamics. Springer, Heidelberg, 2010.

[Pla12a] André Platzer. Logics of dynamical systems. In LICS, pages 13-24, Los Alamitos, 2012. IEEE.

[Pla12b] André Platzer. The structure of differential invariants and differential cut elimination. Log. Meth. Comput. Sci., 8(4:16):1-38, 2012.

[Pla17] André Platzer. A complete uniform substitution calculus for differential dynamic logic. $J$. Autom. Reas., 59(2):219-265, 2017.

[Pla18] André Platzer. Logical Foundations of Cyber-Physical Systems. Springer, Switzerland, 2018.

[PQ08] André Platzer and Jan-David Quesel. KeYmaera: A hybrid theorem prover for hybrid systems. In Alessandro Armando, Peter Baumgartner, and Gilles Dowek, editors, IJCAR, volume 5195 of $L N C S$, pages 171-178, Berlin, 2008. Springer.

[PQ09] André Platzer and Jan-David Quesel. European Train Control System: A case study in formal verification. In Karin Breitman and Ana Cavalcanti, editors, ICFEM, volume 5885 of LNCS, pages 246-265, Berlin, 2009. Springer.

[PT18] André Platzer and Yong Kiam Tan. Differential equation axiomatization: The impressive power of differential ghosts. In Anuj Dawar and Erich Grädel, editors, LICS, New York, 2018. ACM.

$\left[\mathrm{QML}^{+}\right.$16] Jan-David Quesel, Stefan Mitsch, Sarah Loos, Nikos Aréchiga, and André Platzer. How to model and prove hybrid systems with KeYmaera: A tutorial on safety. STTT, 18(1):67-91, 2016.

[SGJ16] Andrew Sogokon, Khalil Ghorbal, and Taylor T Johnson. Non-linear continuous systems for safety verification (benchmark proposal). In ARCH@CPSWeek, volume 43, pages 4251. EasyChair, 2016.

[SGJP16] Andrew Sogokon, Khalil Ghorbal, Paul B. Jackson, and André Platzer. A method for invariant generation for polynomial continuous systems. In Barbara Jobstmann and K. Rustan M. Leino, editors, VMCAI, volume 9583 of LNCS, pages 268-288. Springer, 2016.

[SGS14] Mohamed Amin Ben Sassi, Antoine Girard, and Sriram Sankaranarayanan. Iterative computation of polyhedral invariants sets for polynomial dynamical systems. In $C D C$, pages 6348-6353. IEEE, 2014.

[SV87] Michael A. Savageau and Eberhard O. Voit. Recasting nonlinear differential equations as S-systems: a canonical nonlinear form . Mathematical Biosciences, 87(1):83 - 115, 1987. 
[TPL ${ }^{+}$96] Claire Tomlin, George J. Pappas, John Lygeros, Datta N. Godbole, and Shankar Sastry. Hybrid control models of next generarion air traffic management. In Panos J. Antsaklis, Wolf Kohn, Anil Nerode, and Shankar Sastry, editors, Hybrid Systems IV, volume 1273 of LNCS, pages 378-404. Springer, 1996.

[TPS98] Claire Tomlin, George J. Pappas, and Shankar Sastry. Conflict resolution for air traffic management: a study in multiagent hybrid systems. IEEE Transactions on Automatic Control, 43(4):509-521, Apr 1998.

[WZZ15] Shuling Wang, Naijun Zhan, and Liang Zou. An improved HHL prover: An interactive theorem prover for hybrid systems. In Formal Methods and Software Engineering, pages 382-399. Springer, 2015.

[YJL $\left.{ }^{+} 16\right]$ Gaogao Yan, Li Jiao, Yangjia Li, Shuling Wang, and Naijun Zhan. Approximate bisimulation and discretization of hybrid CSP. In John S. Fitzgerald, Constance L. Heitmeyer, Stefania Gnesi, and Anna Philippou, editors, FM, volume 9995 of LNCS, pages 702-720. Springer, 2016.

[ZHR91] Chaochen Zhou, C.A.R. Hoare, and Anders P. Ravn. A calculus of durations. Information Processing Letters, 40(5):269-276, 1991.

$\left[Z_{L}{ }^{+} 14\right]$ Liang Zou, Jidong Lv, Shuling Wang, Naijun Zhan, Tao Tang, Lei Yuan, and Yu Liu. Verifying Chinese train control system under a combined scenario by theorem proving. In Ernie Cohen and Andrey Rybalchenko, editors, VSTTE 2013, volume 8164 of LNCS, pages 262-280. Springer, 2014.

[ZWR96] Chaochen Zhou, Ji Wang, and Anders P. Ravn. A formal description of hybrid systems. In Rajeev Alur, Thomas A. Henzinger, and Eduardo D. Sontag, editors, Hybrid Systems III, volume 1066 of LNCS, pages 511-530. Springer, 1996.

$\left[Z_{Z}{ }^{+} 13\right]$ Liang Zou, Naijun Zhan, Shuling Wang, Martin Fränzle, and Shengchao Qin. Verifying Simulink diagrams via a Hybrid Hoare Logic prover. In EMSOFT, pages 1-10. IEEE Press, 2013.

[ZZWF15] Liang Zou, Naijun Zhan, Shuling Wang, and Martin Fränzle. Formal verification of Simulink/Stateflow diagrams. In ATVA, volume 9346 of $L N C S$, pages 464-481. Springer, 2015.

\section{A Specification of Machines}

\section{A.1 $\mathrm{M}_{\mathrm{k}}$}

- Processor: Intel Xeon E5-1650 v2 @ 3.5GHz x 6

- Memory: 32GB

- Average CPU Mark on www.cpubenchmark.net: 12695 (full), 1990 (single thread)

\section{A.2 $\mathrm{M}_{\mathrm{hhl}}$}

- Processor: Intel Core i7-4790 CPU @ 3.6GHz

- Memory: 16GB

- Average CPU Mark on www.cpubenchmark.net: 9995 (full), 2284 (single thread) 